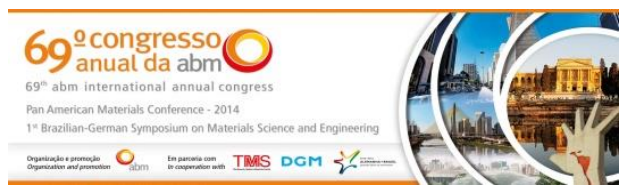

Tema: Metalurgia física e comportamento de materiais em temperaturas elevadas

\title{
AVALIAÇÃO DO COMPORTAMENTO TERMOMECÂNICO DO ACO API 5L X80M - PSL2 MICROLIGADO AO NB-TI ATRAVÉS DE ENSAIOS ISOTÉRMICOS DE TORÇÃO A QUENTE E DE MODELOS MATEMÁTICOS MICROESTRUTURAIS*
}

Marcelo Lucas Pereira Machado Fernanda Huebra² José Brunoro

Resumo

Luciana Nogueira

Os aços APlpossuem as combinações desejáveis de resistência à tração, ductilidade, tenacidade e soldabilidade. A ArcelorMittal Tubarão iniciou 0 desenvolvimento de bobinas a quente do grau API $5 \mathrm{~L}$ X80M-PSL2. O comportamento dos aços está relacionado aos parâmetros de temperatura, deformação, taxa de deformação, taxa de resfriamento e tempo entre passes na laminação a quente. Foi feito um estudo do comportamento termomecânico deste aço através de ensaios de torção a quente isotérmicos e comparados os resultados com os modelos matemáticos microestruturais da literatura ajustados para o aço em questão. Determinau-se a tensão e deformação de pico, tensão e deformação crítica e o amaciamento do material e compados com os modelos utilizados.

Palavras-chave: Ensaio de torção a quente; API 5L X80M - PSL2; Recristalização dinâmica; Modelos microestruturais.

\section{EVALUATION OF THERMOMECHANICAL BEHAVIOR OF THE STEEL API 5L X80M - PSL2 MICROALLOYED NB-TI BY ISOTHERMICAL HOT TORSION TESTS AND MATHEMATICAL MICROSTRUCTURAL MODELS}

\section{Abstract}

The API steels possessing the desirable combinations of tensile strength, ductility, toughness and weldability. The ArcelorMittal Tubarão began development of hot rolled coils grade API 5L PSL2 X80M. The behavior of steels is related to the processing parameters such as temperature, strain, strain rate, cooling rate and interpass time. A study of the thermomechanical behavior of thissteel was done through isothermal hot torsion testing and results were compared with the microstructural models from literature adjusted for this steel. The peak stress and strain, critical strain and stress, and the softening of the material were determined and compared with the models used.

Keywords: Hot torsion; Rolling mill; API 5L X80M - PSL2; Dynamic recrystallization; Microstructural models.

1 Professor Doutor, Titular do Departamento de Engenharia Metalúrgica e de Materiais, IFES, Vitória, ES, Brasil.

2 Engenheira Mecânica, Analista em Assistência Técnica a Clientes da ArcelorMittal Tubarão, Aluna de Mestrado em Engenharia Metalúrgica e de Materiais do IFES, Vitória, ES, Brasil.

3 Tecnólogo em Mecânica, Professor da Coordenadoria, Curso Técnico em Mecânica, Aluno do Programa de Pós-Graduação em Engenharia Metalúrgica e de Materiais, IFES, Vitória, ES, Brasil.

4 Bolsista de Iniciação Científica, Estudante do Curso de Eng. Metalúrgica, IFES, Vitória, ES, Brasil.

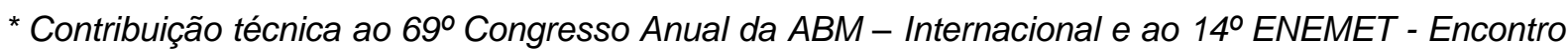
Nacional de Estudantes de Engenharia Metalúrgica, de Materiais e de Minas, 21 a 25 de julho de 2014, São Paulo, SP, Brasil. 


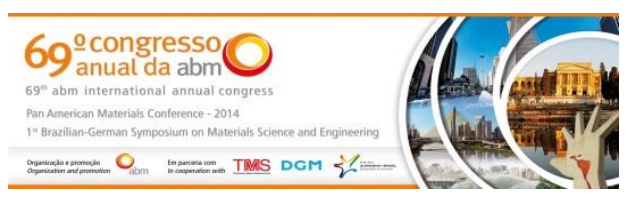

\section{INTRODUÇÃO}

Os principais processos industriais de conformação a quente são a laminação, a extrusão e o forjamento. Esses processos podem ser caracterizados em termos das variáveis relevantes à simulação por torção a quente sendo essas, em geral, a temperatura, a deformação, a taxa de deformação e o tempo entre deformações [1]. $O$ processo de conformação a quente consiste, inicialmente, em aquecer o material até a temperatura de encharque para a austenitização, realizar deformações programadas nas etapas de desbaste e de acabamento e em seguida promover o resfriamento controlado do material. A etapa de acabamento, durante o processo de laminação de tiras a quente, é realizada com curtos tempos de espera entre passes. Sendo o intervalo entre passes pequeno, a recristalização estática poderá não se completar, podendo ter o acúmulo de deformação de um passe para o outro e, consequentemente, o processo passa a ser controlado pela recristalização dinâmica ou metadinâmica. Uma técnica experimental capaz de reproduzir estas condições de processamento e que permite investigar os mecanismos que estão operando é o ensaio de torção a quente, através das curvas de escoamento plástico e do acompanhamento microestrutural. Com ensaios de torção a quente pode-se realizar o ensaio isotérmico impondo parâmetros de processamento tais como a temperatura de reaquecimento, a taxa de resfriamento, a quantidade de deformação e a taxa de deformação.

Pela analise da evolução da curva de escoamento plástico em conjunto com observações microestruturais pode-se determinar o mecanismo de amaciamento dominante em cada uma das etapas de processamento, permitindo assim, determinar os eventos característico da laminação a quente e projetar seqüências de deformações que aperfeiçoam o processamento [2].

A laminação é um exemplo de trabalho mecânico a quente, sendo uma etapa de extrema importância, pois é através dela que se obtêm as formas adequadas dos produtos em aço para uso comercial (chapas, perfis, barras) [3].

\section{MATERIAIS E MÉTODOS}

\subsection{Material Utilizado}

O material utilizado foi um aço API $5 \mathrm{~L}$ X80 (com alto $\mathrm{Nb}$ ) produzido noLaminador de Tiras a Quente da ArcelorMittal Tubarão cuja composição química está apresentada no Tabela 1.

Tabela 1. Composição química do API $5 \mathrm{~L}$ X80 (com alto Nb).

\begin{tabular}{|c|c|c|c|c|c|c|c|c|c|}
\hline \multicolumn{10}{|c|}{ Composição quimica (\% em peso) } \\
\hline $\mathrm{C}$ & $\mathrm{Si}$ & $\mathrm{Mn}$ & $\mathrm{P}$ & $\mathrm{S}$ & $\mathrm{Al}$ & $\mathrm{N}$ & $\mathrm{Nb}$ & $\mathrm{Ti}$ & $\mathrm{Ca}$ \\
\hline$\leq 0,100$ & $\leq 0,300$ & $\leq 1,700$ & $\leq 0,018$ & $\leq 0,005$ & $\leq 0,050$ & $\leq 0,010$ & $\sim 0,090$ & $\leq 0,030$ & $\leq 0,005$ \\
\hline
\end{tabular}

\subsection{Preparação dos Corpos de Prova}

Os Corpos de prova de torção foram usinados a partir de uma chapa de $15,88 \mathrm{~mm} x$ $1.500 \mathrm{~mm} \times 500 \mathrm{~mm}$, com diâmetro útil de $5 \mathrm{~mm}$ e comprimento útil de $20 \mathrm{~mm}$, como representado na Figura 1.

\footnotetext{
* Contribuição técnica ao 69 Congresso Anual da ABM - Internacional e ao 14ํㅡㄹ ENET - Encontro Nacional de Estudantes de Engenharia Metalúrgica, de Materiais e de Minas, 21 a 25 de julho de 2014, São Paulo, SP, Brasil.
} 

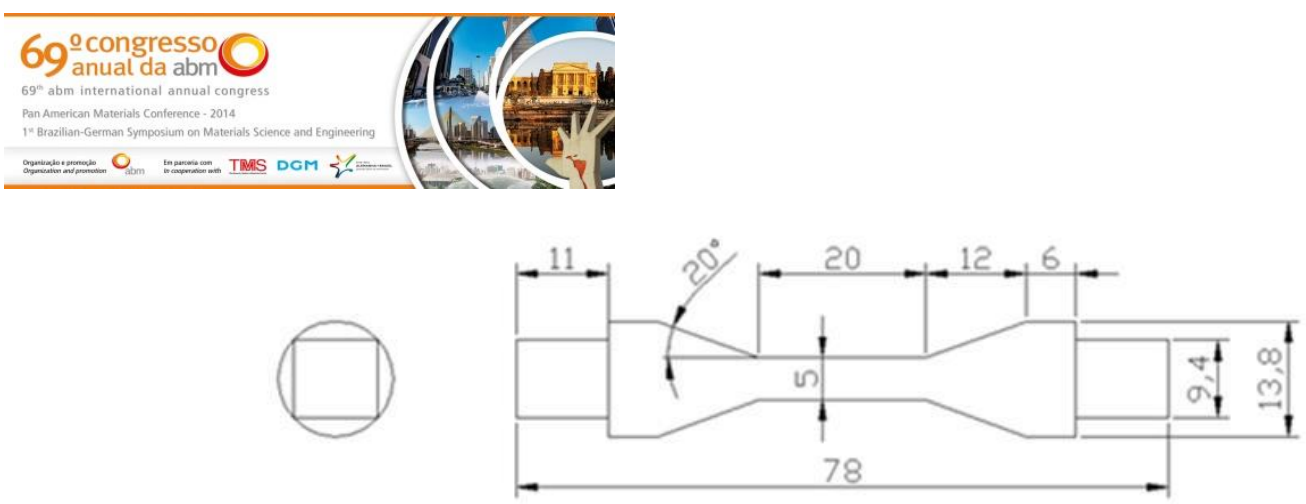

Figura 1 - Desenho esquemático do corpo de prova.

\subsection{Maquina de Torção}

Todos os ensaios foram realizados utilizando-se uma máquina horizontal de torção a quente INSTRON, modelo 55MT do laboratório de conformação mecânica do IFES. Colocou-se um tubo de quartzo de aproximadamente $40 \mathrm{~mm}$ com injeção de argônio e gás $\mathrm{CO}_{2}$ para criar uma atmosfera inerte durante o ensaio, ocasionar o tratamento térmico de têmpera e evitar a corrosão do corpo de prova.

\subsubsection{Ensaios isotérmicos e contínuos}

Os corpos de prova foram aquecidos até a temperatura de $1.240^{\circ} \mathrm{C}$ a uma taxa média de $3^{\circ} \mathrm{C} / \mathrm{s}$ e mantidos nesta temperatura por um período de 3 minutos.A seguir resfriados a uma taxa média de $1^{\circ} \mathrm{C} / \mathrm{s}$ até a temperatura de ensaio e mantidos nesta temperatura por 1 minuto, para eliminação dos efeitos de gradientes térmicos, antes do início da deformação.

Foram realizados dois grupos de ensaios, sendo o primeiro com a taxa de deformação fixa e variando a temperatura e o segundo grupo com a temperatura fixa e variando a taxa de deformação. Grupo 1: as temperaturas utilizadas nos ensaios isotérmicos foram $1.150^{\circ} \mathrm{C}, 1.100^{\circ} \mathrm{C}, 1.000^{\circ} \mathrm{C}, 950^{\circ} \mathrm{C}$ e850 ${ }^{\circ} \mathrm{C}$, a taxa de deformação foi de $0,2 \mathrm{~s}^{-1}$ e a deformação máxima de 3 . Grupo 2: para estudar ocomportamento do material em relação a taxa de deformação foram realizadosensaios na temperatura de $1.150^{\circ} \mathrm{C}$ e as taxas de deformação de $0,2 \mathrm{~s}^{-1} ; 0,4 \mathrm{~s}^{-1}$ e $0,8 \mathrm{~s}^{-1}$, comdeformação máxima de 3 . Na Figura 2 está mostrado esquematicamente o ciclo termomecânico destes ensaios isotérmicos.

\section{RESULTADOS E DISCUSSÃO}

\subsection{Ensaios Isotérmicos Contínuos}

Na Figura 3 podemos observar o comportamento de um aço API $5 \mathrm{~L}$ X80 submetido a uma taxa de deformação de $0,2 \mathrm{~s}^{-1}$ para diferentes temperaturas. Observa-se um aumento na tensão de pico à medida que a temperatura decresce. Esse resultado mostra similaridade com outros estudos realizados através de ensaio de torção a quente [4-6]. Esse comportamento é esperado visto que a densidade e distribuição de discordâncias, assim como a energia armazenada na deformação são fatores que dependem diretamente da temperatura durante a deformação do metal $[7,8]$. Com o decréscimo da temperatura a mobilidade das discordâncias diminui, gerando um aumento da tensão e um deslocamento das curvas para a esquerda, o que mostra que existe um maior encruamento do material $[4,5]$.

\footnotetext{
* Contribuição técnica ao $69^{\circ}$ Congresso Anual da ABM - Internacional e ao 14ํㅡㄹ ENEMET - Encontro Nacional de Estudantes de Engenharia Metalúrgica, de Materiais e de Minas, 21 a 25 de julho de 2014, São Paulo, SP, Brasil.
} 

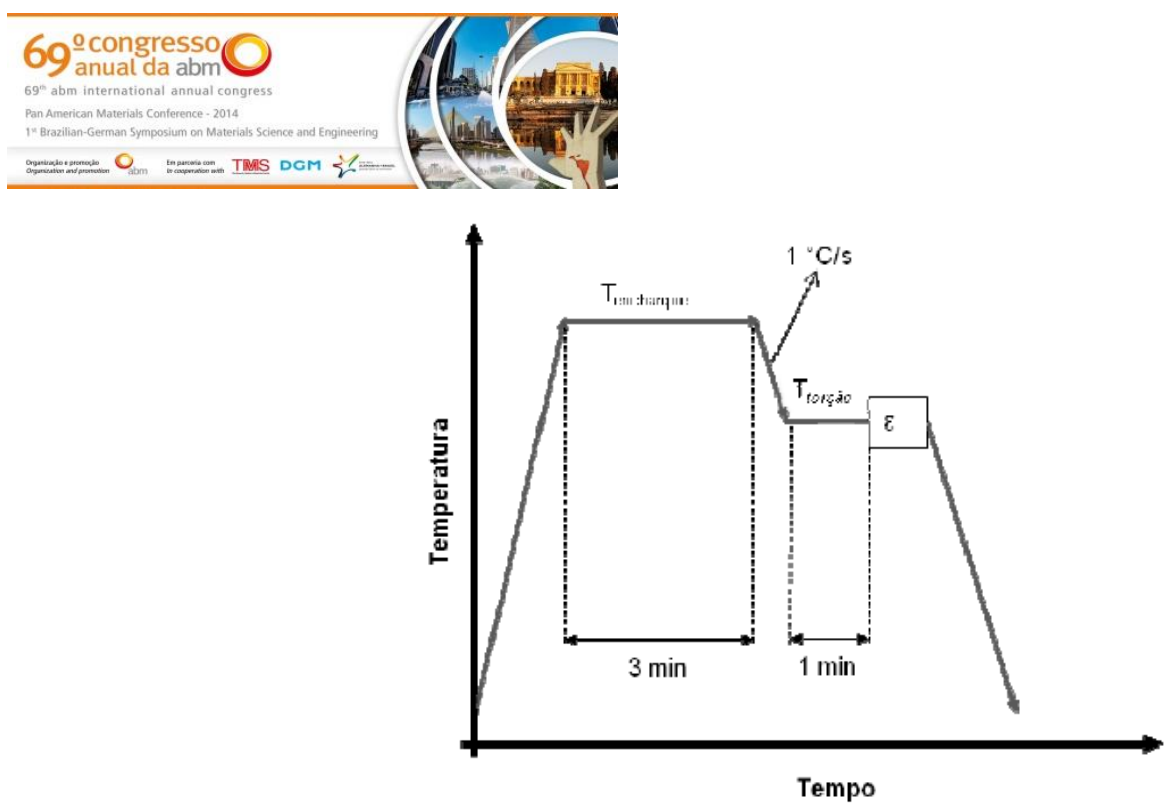

Figura 2 - Representação esquemática do ciclo termomecânico utilizado nos ensaios isotérmicos.

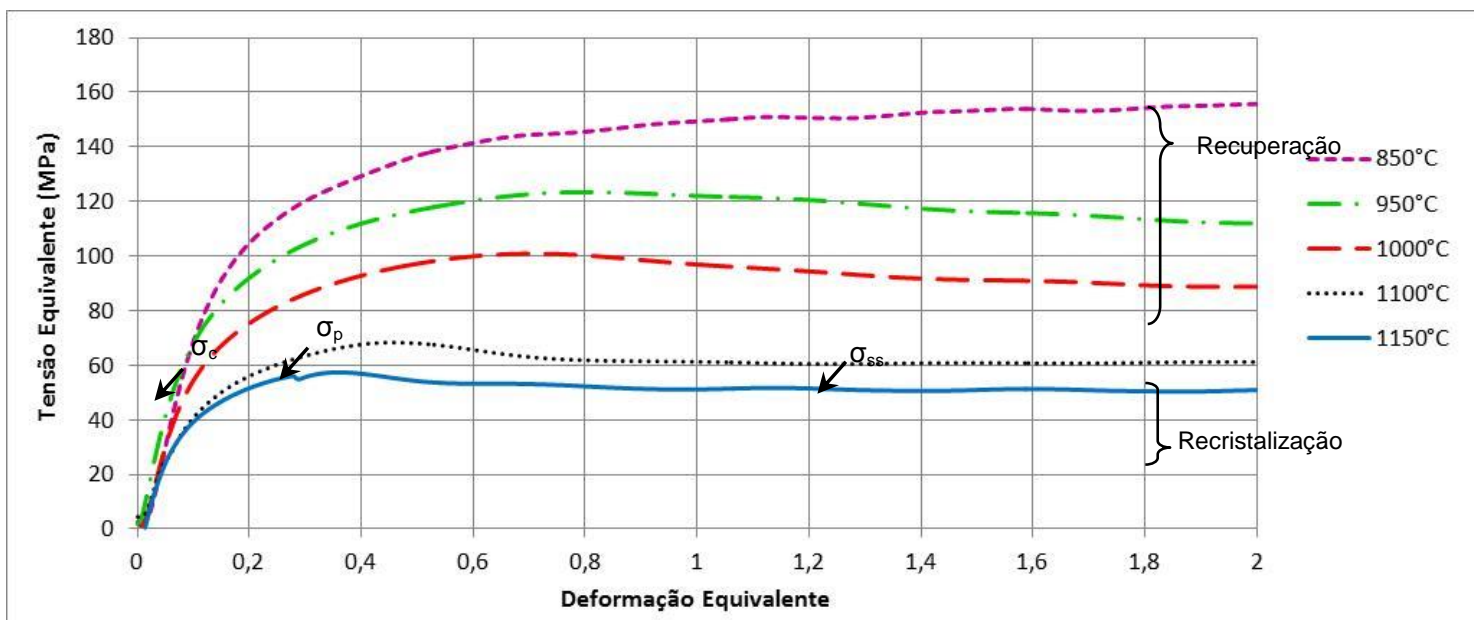

Figura 3 - Curvas Tensão equivalente versus deformação equivalente obtidas por ensaios de torção a quente para um aço API $5 \mathrm{~L}$ X80 a diferentes temperaturas e taxa de deformação de $0,2 \mathrm{~s}^{-1}$.

Observamos que nas temperaturas de $1.150^{\circ} \mathrm{C}$ e $1.100^{\circ} \mathrm{C}$ as curvas de escoamento plástico apresentam uma forma típica de materiais que se recristalizam dinamicamente, ou seja, inicialmente a tensão de escoamento plástico aumenta com a deformação até um valor máximo e em seguida seu valor diminui até um estado estacionário. Esse comportamento era esperado, visto que essas temperaturas estão acima da temperatura de não recristalização da austenita, que neste estudo foi obtida em outro ensaio e o seu valor foi de $1.050^{\circ} \mathrm{C}$. Nas curvas correspondentes as temperaturas de 1.150 e $1.100^{\circ} \mathrm{C}$ estão identificadas a tensão crítica para inicio da recristalização, a tensão de pico $\left(\sigma_{p}\right)$ e a tensão no estado estacionário ( $\left.\sigma_{s s}\right)$.

Para as temperaturas de $1.000^{\circ} \mathrm{C}, 950^{\circ} \mathrm{C}$ e $850^{\circ} \mathrm{C}$ as curvas apresentam uma forma típica de materiais que se recuperam dinamicamente, isto é, acontece um maior encruamento no início da deformação até se atingir o pico de tensão. Quanto mais mais baixas são as temperaturas maior é a ocorrência de encruamento e por isso a recuperação do metal se torna mais lenta.

Através das curvas de Taxa de Encruamento $(\theta)$ versus Tensão Equivalente $(\sigma)$ ou Derivada da Taxa de Encruamento $(\mathrm{d} \theta / \mathrm{d} \sigma)$ versus Tensão Equivalente $(\sigma)$ é possível determinar a deformação crítica $(\varepsilon c)$ para o início da recristalização dinâmica.

Na Figura 4 estão mostradas as curvas da taxa de encruamento $(\theta)$ versus tensão equivalente $(\sigma)$ para ensaios realizados com taxa de deformação de $0,2 \mathrm{~s}^{-1}$ para temperaturas de deformação acima da $\mathrm{Tr}$ r.

* Contribuição técnica ao $69^{\circ}$ Congresso Anual da ABM - Internacional e ao 14ํㅡㄹ ENEMET - Encontro Nacional de Estudantes de Engenharia Metalúrgica, de Materiais e de Minas, 21 a 25 de julho de 2014, São Paulo, SP, Brasil. 

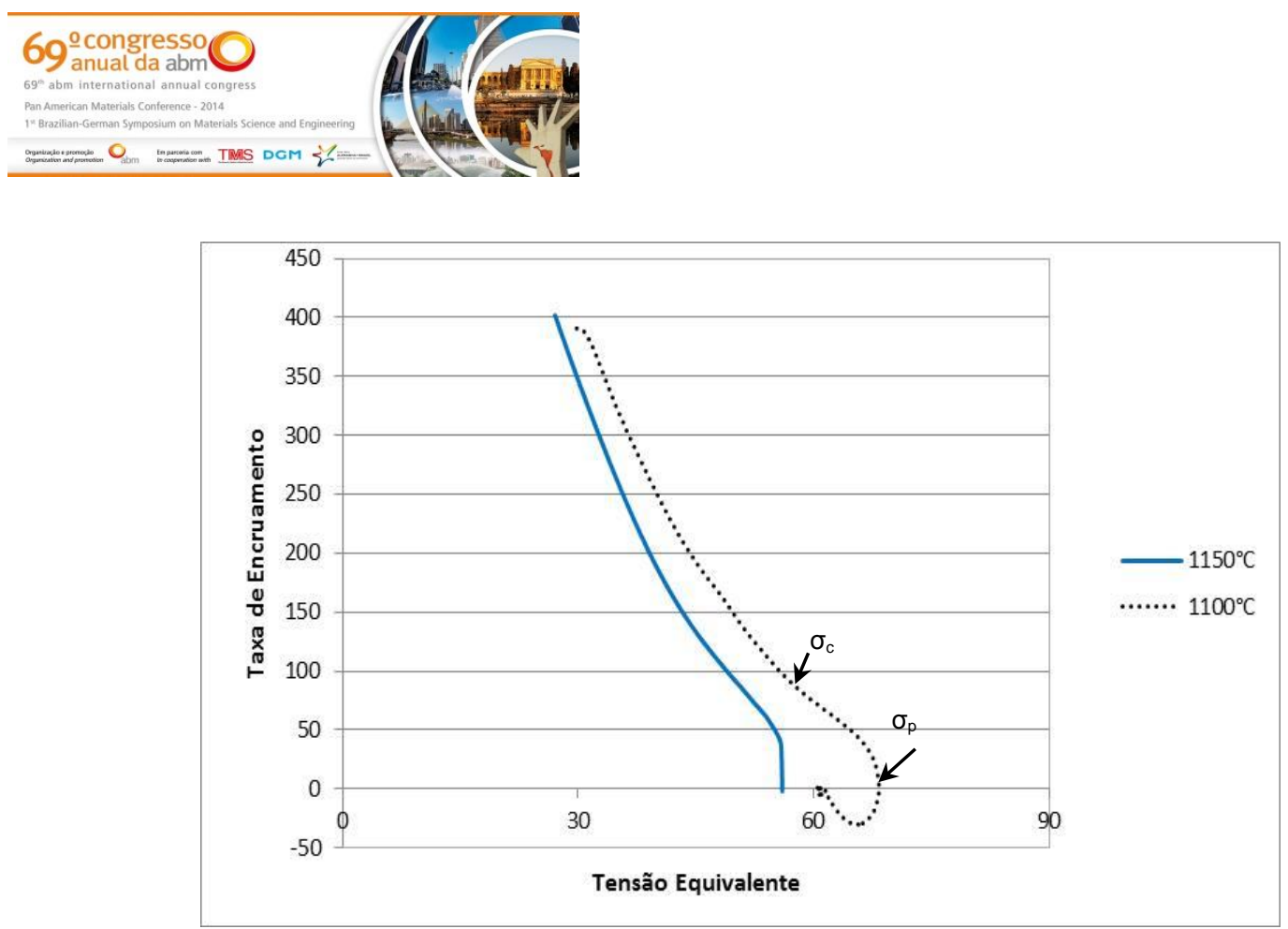

Figura 4 - Variação da Taxa de Encruamento $(\theta)$ versus a tensão equivalente para um aço API $5 \mathrm{~L}$ X80 a diferentes temperaturas a taxa de deformação constante de $0,2 \mathrm{~s}^{-1}$.

Observamos nas curvas da Figura 4 que a taxa de encruamento diminui com 0 aumento da tensão inicialmente de uma forma linear e em seguida de uma forma aproximadamente parabólica, conforme esperado. Esta mudança no comportamento das curvas se deve ao acúmulo de discordâncias e surgimento de subgrãos.

Na região parabólica das curvas observa-se um ponto de inflexão que corresponde a tensão crítica $\left(\sigma_{c}\right)$ para o início da recristalização dinâmica. Para determinar a deformação crítica $(\varepsilon c)$ transporta-se o valor da tensão crítica $\left(\sigma_{c}\right)$ para a curva de tensão versus deformação e encontra-se a deformação crítica $(\varepsilon c)$.

Ainda na Figura 4 observa-se que a taxa de encruamento $(\theta)$ decresce até um valor de tensão máxima, que corresponde a tensão de pico $\left(\sigma_{p}\right)$, onde a taxa de encruamento $(\theta)$ é igual a zero.

Para uma melhor visualização foram identificadas a tensão crítica $\left(\sigma_{c}\right)$ e tensão de pico $\left(\sigma_{p}\right)$ na curva correspondente a temperatura de $1.100^{\circ} \mathrm{C}$.

Outra forma de encontrar a deformação crítica $(\varepsilon c)$ é através da curva da Derivada da Taxa de Encruamento $(\mathrm{d} \theta / \mathrm{d} \sigma)$ versus Tensão Equivalente $(\sigma)$. Na Figura 5 estão mostradas as curvas da derivada da taxa de encruamento $(\mathrm{d} \theta / \mathrm{d} \sigma)$ versus tensão equivalente $(\sigma)$ para ensaios realizados com taxa de deformação de $0,2 \mathrm{~s}^{-1}$.

O ponto máximo da curva representa a tensão crítica $\left(\sigma_{c}\right)$, que é usada para determinar a deformação crítica $\left(\varepsilon_{c}\right)$ por correspondência na curva de escoamento plástico $(\sigma \times \varepsilon)$.

A partir das curvas das Figuras 3, 4 e 5 foi organizada a Tabela 2 com os parâmetros termomecânicos encontrados, como tensão de pico $\left(\sigma_{p}\right)$, tensão crítica $\left(\sigma_{c}\right)$, deformação de pico $\left(\varepsilon_{p}\right)$, deformação crítica $\left(\varepsilon_{c}\right)$ e tensão no estado estacionário $\left(\sigma_{s s}\right)$ para taxa de deformação a $0,2 \mathrm{~s}^{-1}$ variando a temperatura.

* Contribuição técnica ao 69 Congresso Anual da ABM - Internacional e ao 14ํㅡㄹ ENET - Encontro Nacional de Estudantes de Engenharia Metalúrgica, de Materiais e de Minas, 21 a 25 de julho de 2014, São Paulo, SP, Brasil. 

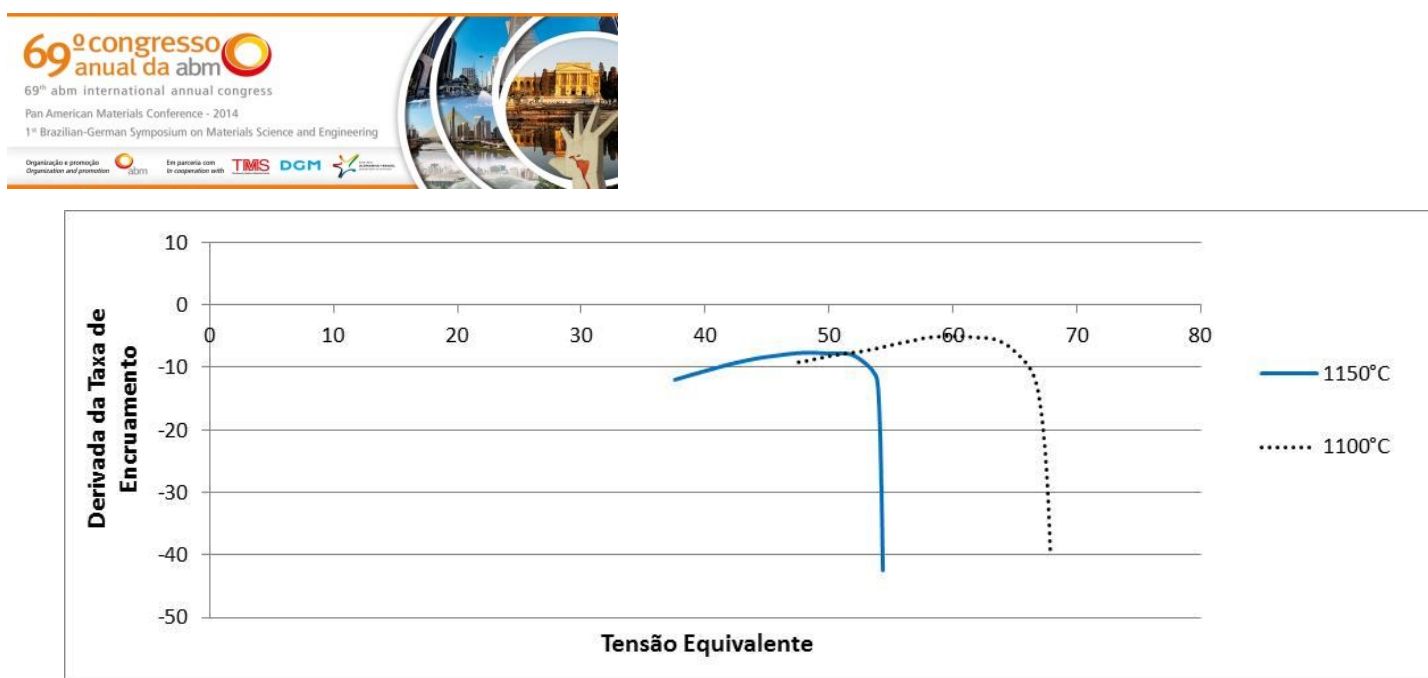

Figura 5 - Curvas da Derivada da Taxa de Encruamento $(d \theta / d \sigma)$ versus a tensão equivalente $(\sigma)$ para um aço API 5L X80 a diferentes temperaturas e taxa de deformação constante de 0,2s-1.

Tabela 2 - Valores obtidos nos ensaios isotérmicos para um aço API $5 \mathrm{~L}$ X80 a diferentes temperaturas e taxa de deformação constante de $0,2 \mathrm{~s}-1$

\begin{tabular}{ccccccc}
\hline $\begin{array}{c}\text { Temperatura } \\
(\mathbf{o} \text { C) }\end{array}$ & $\begin{array}{c}\sigma_{\mathrm{p}} \\
(\mathbf{M P a})\end{array}$ & $\begin{array}{c}\sigma_{\mathrm{c}} \\
(\mathrm{MPa})\end{array}$ & $\boldsymbol{\varepsilon}_{\mathrm{p}}$ & $\boldsymbol{\varepsilon}_{\mathrm{c}}$ & $\boldsymbol{\varepsilon}_{\mathrm{c}} / \boldsymbol{\varepsilon}_{\mathrm{p}}$ & $\begin{array}{c}\sigma_{\mathrm{ss}} \\
(\mathrm{MPa})\end{array}$ \\
\hline $\mathbf{1 . 1 5 0}$ & 57,4 & 48,5 & 0,36 & 0,17 & 0,47 & 50,5 \\
$\mathbf{1 . 1 0 0}$ & 68,3 & 59,7 & 0,45 & 0,24 & 0,53 & 61,1 \\
$\mathbf{1 . 0 0 0}$ & 100,9 & - & 0,70 & - & - & 88,6 \\
$\mathbf{9 5 0}$ & 123,4 & - & 0,80 & - & - & 107,0 \\
$\mathbf{8 5 0}$ & 155,6 & - & 1,99 & - & - & 155,6 \\
\hline
\end{tabular}

De acordo com a Tabela 1 os valores de tensão de pico e tensão crítica aumentam com o decréscimo de temperatura como esperado.

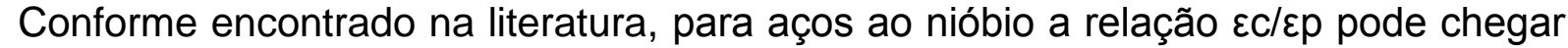
a 0,5 [8]. Para as temperaturas acima da $\mathrm{Tnr}, 1.150^{\circ} \mathrm{C}$ e $1.100^{\circ} \mathrm{C}$, os valores encontrados para relação $\varepsilon c / \varepsilon p$ foram respectivamente 0,47 e 0,53 conforme esperado. Na Figura 6 podemos observar o comportamento de um aço API 5L X80 submetido ao ensaio de torção a quente em diferentes taxas de deformação e a temperatura de $1.150^{\circ} \mathrm{C}$.

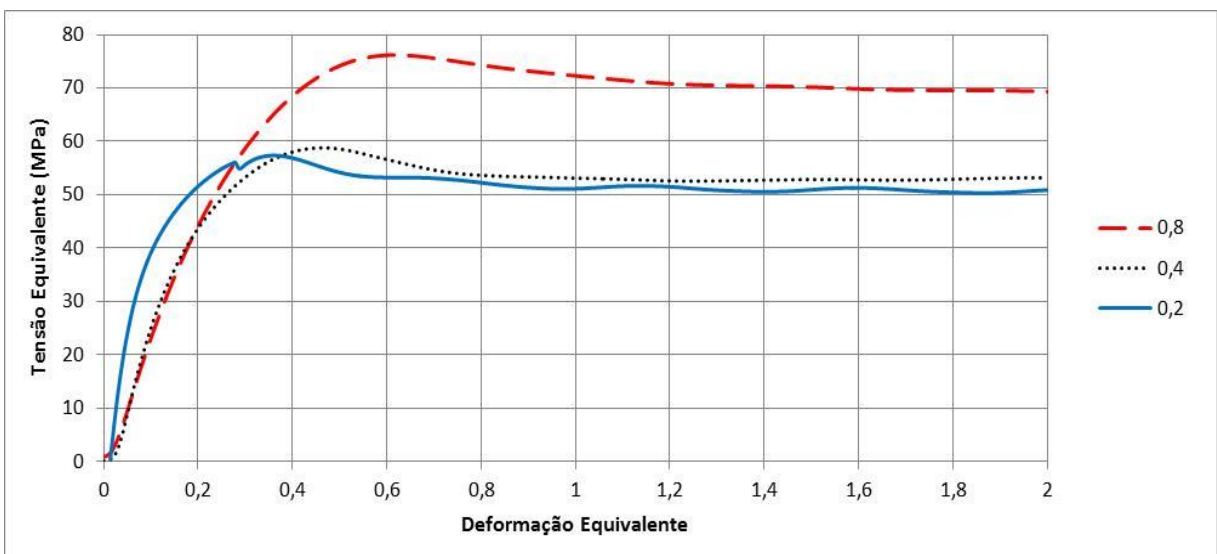

Figura 6 - Curvas Tensão equivalente versus deformação equivalente obtidas por ensaios de torção a quente para um aço API $5 \mathrm{~L}$ X80 em diferentes taxas de deformação e a temperatura de $1.150^{\circ} \mathrm{C}$.

$\mathrm{Na}$ Figura 6 observa-se que as três curvas resultantes dos ensaios a diferentes taxas de deformação e temperatura de $1.150^{\circ} \mathrm{C}$ apresentam uma forma típica de materiais que se recristalizam dinamicamente. Esse comportamento era esperado, visto que a temperatura do ensaio está acima da temperatura de não recristalização da austenita $\left(1.050^{\circ} \mathrm{C}\right)$.

* Contribuição técnica ao $69^{\circ}$ Congresso Anual da ABM - Internacional e ao $14^{\circ}$ ENEMET - Encontro Nacional de Estudantes de Engenharia Metalúrgica, de Materiais e de Minas, 21 a 25 de julho de 2014, São Paulo, SP, Brasil. 
Podemos observar que quanto maior a taxa de deformação as curvas tensão versus deformação tendem a se deslocar para cima e para direita. Concluímos, então, que quanto maior a taxa de deformação aplicada maior será a tensão de pico do aço.

Para ajudar na compreensão do comportamento do aço em relação às diferentes taxas de deformação podemos verificar a tensão em função do tempo de duração dos testes, conforme mostrado na Figura 7. Dessa forma podemos observar o comportamento da tensão como se os testes fossem realizados simultaneamente.

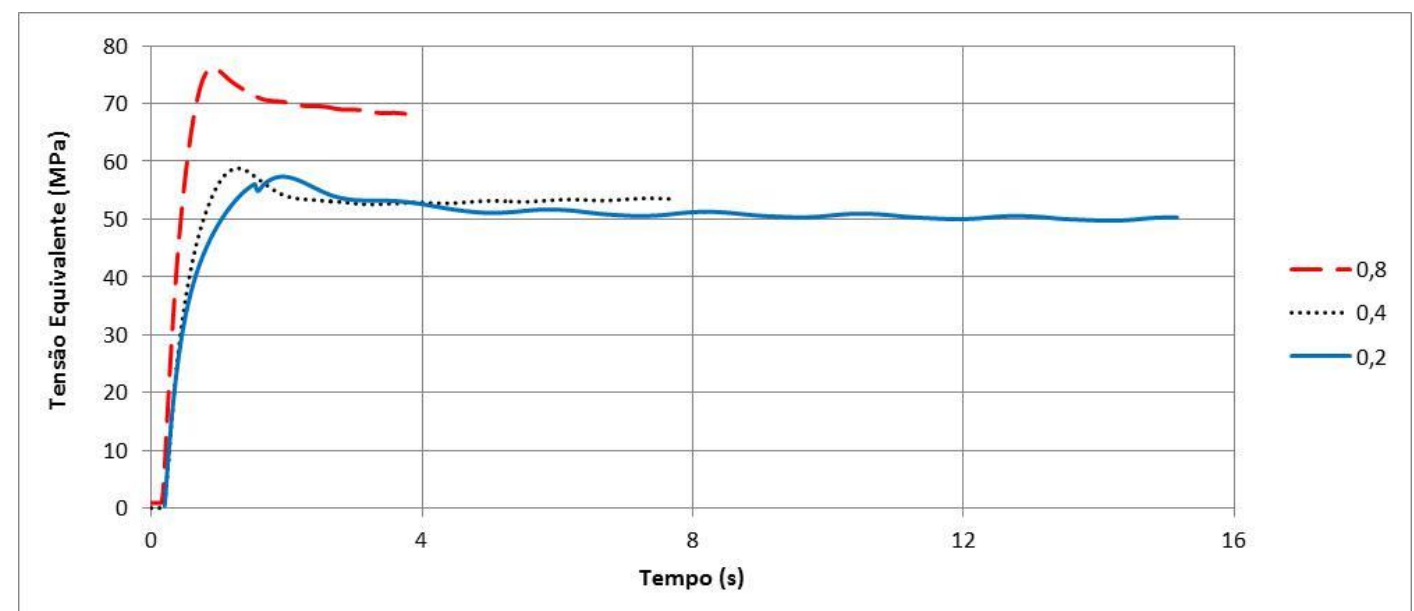

Figura 7 - Curvas Tensão equivalente versus tempo de deformação obtidas por ensaios de torção a quente para um aço API $5 \mathrm{~L}$ X80 a diferentes taxas de deformação a temperatura de $1.150^{\circ} \mathrm{C}$.

Observamos que o tempo gasto para a realização do ensaio com a aplicação da mesma deformação é menor quanto maior a taxa de deformação utilizada, devido a geração de grande quantidade de discordâncias num tempo menor, levando a ocorrência da tensão crítica mais cedo. Outra observação que pode ser feita é que a tensão se eleva mais rapidamente para taxas de deformação maiores do que em menores taxas de deformação.

$\mathrm{Na}$ Figura 8 estão mostradas as curvas da taxa de encruamento $(\theta)$ versus tensão equivalente $(\sigma)$ para ensaios realizados a diferentes taxas de deformação e temperatura de $1.150^{\circ} \mathrm{C}$. Podemos observar que para taxa de deformação maior $\left(0,8 \mathrm{~s}^{-1}\right)$ não foi observado o ponto de inflexão conforme mostrado na figura 9 . Esse fato também foi observado por [6] para uma mesma taxa de deformação e temperatura de $1.050^{\circ} \mathrm{C}$ para um aço SAE 1518. Este comportamento pode ser explicado pela alta taxa de deformação, que tende a gerar mais discordâncias em um curto espaço de tempo, o que dificulta a percepção do ponto de inflexão na curva. A influência da recristalização fica melhor evidenciada a taxas de deformações menores $\left(0,2\right.$ e $\left.0,4 \mathrm{~s}^{-1}\right)$. Esse fato também pode ser observado nas curvas da derivada da taxa de encruamento $(\mathrm{d} \theta / \mathrm{d} \sigma)$ versus tensão equivalente $(\sigma)$, Figura 9.

* Contribuição técnica ao $69^{\circ}$ Congresso Anual da ABM - Internacional e ao 14ํㅡㄹ ENEMET - Encontro Nacional de Estudantes de Engenharia Metalúrgica, de Materiais e de Minas, 21 a 25 de julho de 2014, São Paulo, SP, Brasil. 

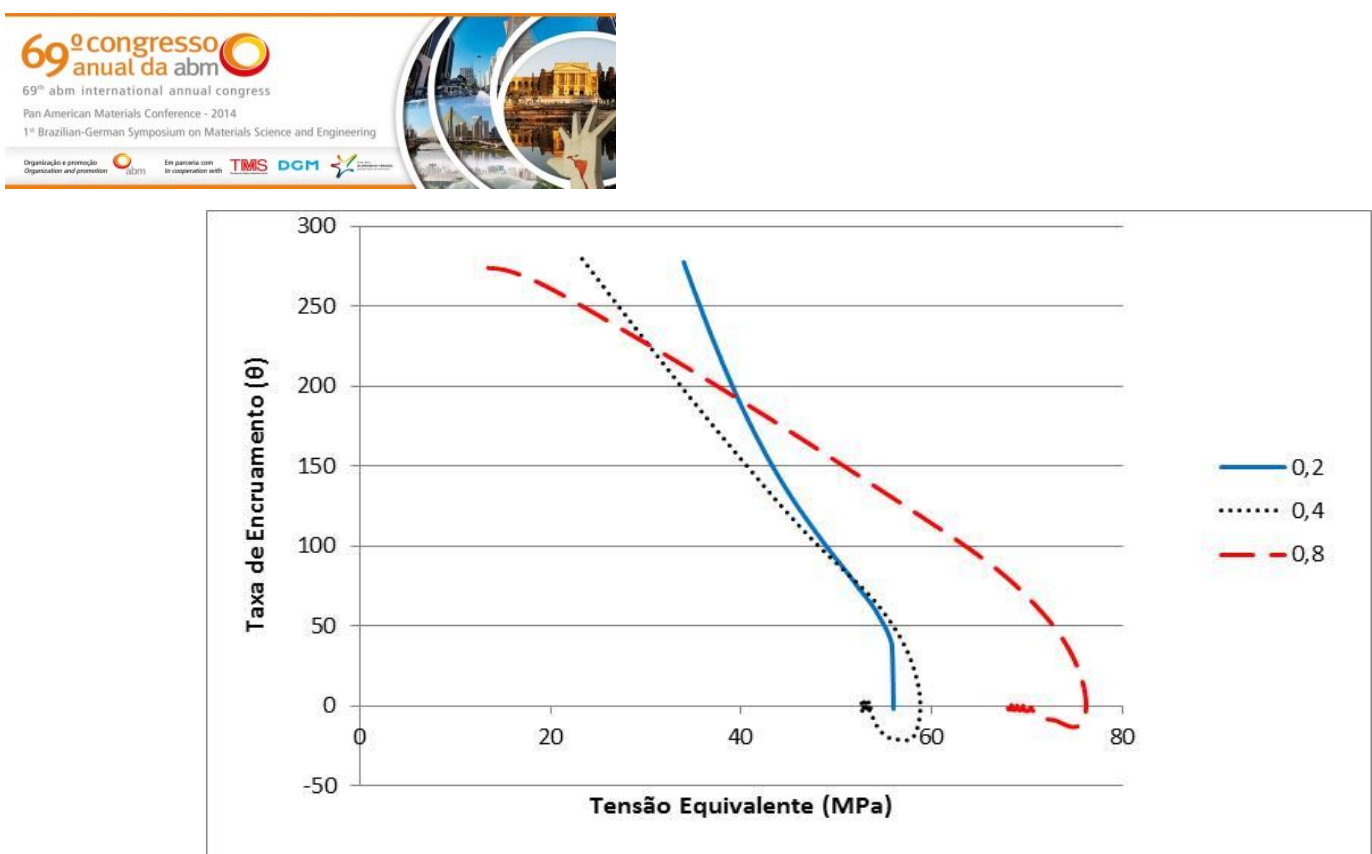

Figura 8 - Variação da Taxa de Encruamento $(\theta)$ versus a tensão equivalente para um aço API $5 \mathrm{~L}$ X80 a diferentes taxas de deformação e temperatura de $1.150^{\circ} \mathrm{C}$.

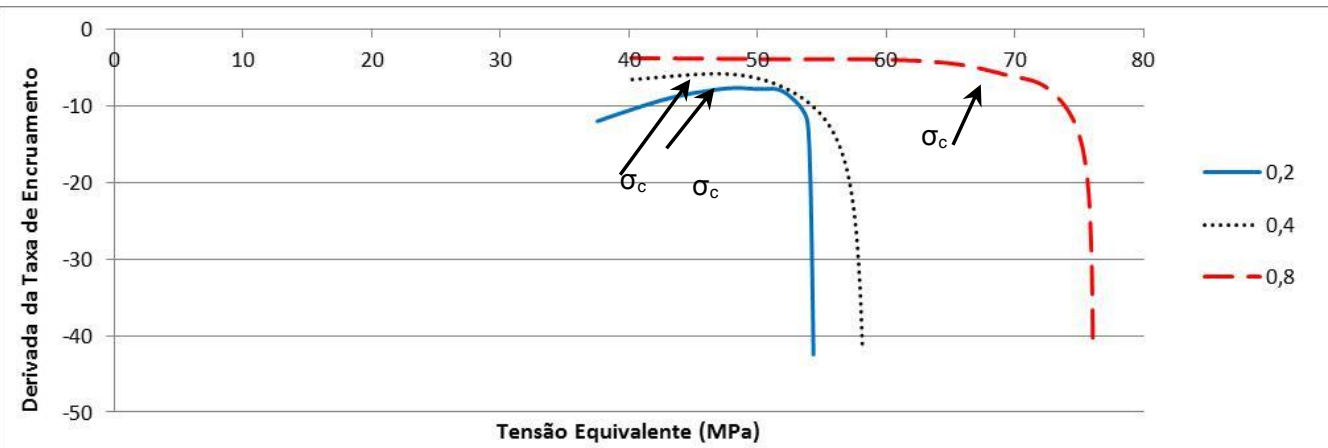

Figura 9 - Curvas da Derivada da Taxa de Encruamento $(d \theta / d \sigma)$ versus a tensão equivalente $(\sigma)$ para um aço API $5 \mathrm{~L}$ X80 a diferentes taxas de deformação e temperatura de 1.150ㄷ․

Nas curvas da Figura 9 para taxas de deformações menores $\left(0,2\right.$ e $\left.0,4 \mathrm{~s}^{-1}\right)$ é possível identificar a tensão crítica $\left(\sigma_{c}\right)$ através do ponto de máximo das curvas. Já na curva referente a taxa de deformação de $0,8 \mathrm{~s}^{-1}$ é mais difícil identificar a tensão crítica $\left(\sigma_{c}\right)$, pois não há presença de pico.

A partir das curvas das Figuras 7,8 e 9 foi organizada a Tabela 2 com os parâmetros termomecânicos encontrados, como tensão de pico $\left(\sigma_{p}\right)$, tensão crítica $\left(\sigma_{c}\right)$, deformação de pico $\left(\varepsilon_{p}\right)$, deformação crítica $\left(\varepsilon_{c}\right)$ e tensão no estado estacionário $\left(\sigma_{\mathrm{ss}}\right)$ para deformações de 0,$2 ; 0,4$ e $0,8 \mathrm{~s}^{-1}$ e uma temperatura de $1.150^{\circ} \mathrm{C}$.

Tabela 2 - Valores obtidos nos ensaios isotérmicos para um aço API $5 \mathrm{~L}$ X80 a diferentes taxas de deformação e temperatura de $1.150^{\circ} \mathrm{C}$.

\begin{tabular}{ccccccc}
\hline$\dot{\varepsilon}\left(\mathbf{s}^{-1}\right)$ & $\begin{array}{c}\sigma_{\mathbf{p}} \\
(\mathbf{M P a})\end{array}$ & $\begin{array}{c}\boldsymbol{\sigma}_{\mathbf{c}} \\
(\mathbf{M P a})\end{array}$ & $\boldsymbol{\varepsilon}_{\mathbf{p}}$ & $\boldsymbol{\varepsilon}_{\mathrm{c}}$ & $\boldsymbol{\varepsilon}_{\mathrm{c}} / \varepsilon_{\mathbf{p}}$ & $\begin{array}{c}\sigma_{\mathbf{s s}} \\
(\mathbf{M P a})\end{array}$ \\
\hline $\mathbf{0 , 2}$ & 57,4 & 48,5 & 0,36 & 0,17 & 0,47 & 50,5 \\
$\mathbf{0 , 4}$ & 58,8 & 49,8 & 0,46 & 0,23 & 0,50 & 53,1 \\
$\mathbf{0 , 8}$ & 76,2 & 64,1 & 0,62 & 0,35 & 0,57 & 68,3 \\
\hline
\end{tabular}

De acordo com a Tabela 2 os valores de tensão de pico e da tensão crítica aumentam com o aumento da taxa de deformação e os valores encontrados para relação $\varepsilon_{c} / \varepsilon_{p}$ foram próximos a 0,5 como era esperado.

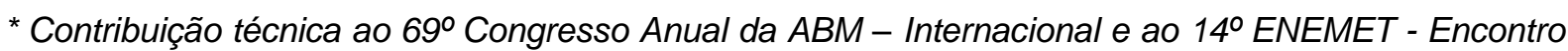
Nacional de Estudantes de Engenharia Metalúrgica, de Materiais e de Minas, 21 a 25 de julho de 2014, São Paulo, SP, Brasil. 


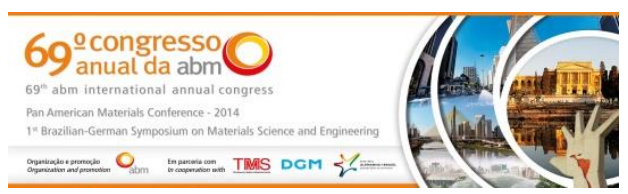

\subsection{Parâmetros Termomecânicos (Experimentais Versus Modelo)}

$\mathrm{Na}$ Tabela 3 mostramos os valores da $\varepsilon_{\mathrm{c}}, \quad \varepsilon_{\mathrm{p}}, \quad \varepsilon_{\mathrm{c}} / \varepsilon_{\mathrm{p}}$ e $\sigma_{\mathrm{ss}}$ encontradas experimentalmente e através de equações da literatura para aços ao $\mathrm{Nb}$.

Conforme pode ser visto a relação $\varepsilon_{c} / \varepsilon_{p}$ encontrada através das equações da literatura foi de aproximadamente 0,5 assim como os valores encontrados experimentalmente. Esses valores estão dentro do esperado para aços ao $\mathrm{Nb}$. Porém os valores encontrados através das equações da literatura para $\varepsilon_{c}$ e $\varepsilon_{p}$ estão bem acima dos valores encontrados experimentalmente.

Tabela 3 - Valores de $\varepsilon c$, $\varepsilon p$ e oss obtidos nos ensaios isotérmicos para um aço API $5 \mathrm{~L}$ X80 e através de equações da literatura para aços ao $\mathrm{Nb}$ para diferentes temperaturas e diferentes taxas de deformação.

\begin{tabular}{|c|c|c|c|c|c|c|c|c|c|}
\hline \multirow[b]{2}{*}{$\mathrm{T}\left({ }^{\circ} \mathrm{C}\right)$} & \multirow[b]{2}{*}{$\dot{\varepsilon}\left(\mathbf{s}^{-1}\right)$} & \multicolumn{4}{|c|}{ Experimentais } & \multicolumn{4}{|c|}{ Equações da literatura para aço ao $\mathrm{Nb}$} \\
\hline & & $\varepsilon_{\mathrm{c}}$ & $\varepsilon_{\mathrm{p}}$ & $\varepsilon_{c} / \varepsilon_{p}$ & $\begin{array}{c}\sigma_{\mathrm{ss}} \\
(\mathrm{MPa})\end{array}$ & $\begin{array}{c}\varepsilon_{c} \\
(\text { Eq. 3.4) }\end{array}$ & $\begin{array}{c}\varepsilon_{p} \\
(E q .3 .1)\end{array}$ & $\varepsilon_{\mathrm{c}} / \varepsilon_{\mathrm{p}}$ & $\begin{array}{c}\sigma_{\mathrm{ss}} \\
(\mathrm{MPa}) \\
(\mathrm{Eq} .3 .3)\end{array}$ \\
\hline 1.150 & 0,2 & 0,17 & 0,36 & 0,47 & 50,5 & 0,33 & 0,67 & 0,49 & 63,0 \\
\hline 1.150 & 0,4 & 0,23 & 0,46 & 0,50 & 53,1 & 0,37 & 0,76 & 0,49 & 67,0 \\
\hline 1.150 & 0,8 & 0,35 & 0,62 & 0,57 & 68,3 & 0,42 & 0,85 & 0,49 & 71,4 \\
\hline 1.100 & 0,2 & 0,24 & 0,45 & 0,53 & 61,1 & 0,40 & 0,82 & 0,49 & 69,9 \\
\hline 1.000 & 0,2 & - & 0,70 & - & 88,6 & - & 1,27 & - & 88,2 \\
\hline 950 & 0,2 & - & 0,80 & - & 107,0 & - & 1,62 & - & 100,4 \\
\hline 850 & 0,2 & - & 1,99 & - & 155,6 & - & 2,83 & - & 135,0 \\
\hline
\end{tabular}

Na equação 3.1 desenvolvida por [9] para descrever a $\varepsilon_{\mathrm{p}}$ foi proposto para o aço API $5 \mathrm{~L}$ X80 com alto teor de nióbio um ajuste no valor da constante de 2,8 para 1,54 com o objetivo de adequar os valores encontrados através da equação ao resultado encontrado experimentalmente, como mostrado na equação 3.2. Consequente os valores da $\varepsilon_{c}$ foram ajustados, uma vez que a equação para $\varepsilon_{c}$ proposta por [9] (1996) depende da $\varepsilon_{p}$.

$$
\begin{aligned}
& \varepsilon_{\mathrm{p}}=\frac{(1+20 \mathrm{Nb})}{1,78} \cdot 2,8 \cdot 10^{-4} \cdot \mathrm{d}_{\mathrm{i}-1}^{0,5} \cdot(\mathrm{z})^{0,17} \\
& \varepsilon_{\mathrm{p}}=\frac{(1+20 \mathrm{Nb})}{1,78} \cdot 1,54 \cdot 10^{-4} \cdot \mathrm{d}_{\mathrm{i}-1}^{0,5} \cdot(\mathrm{z})^{0,17}
\end{aligned}
$$

$\mathrm{Na}$ Figura 10 mostramos as tensões no regime estacionário (oss) encontradas experimentalmente (Tabela 2Tabela 3) em função do parâmetro Zener-Hollomon (equação 3.30). Foram traçadas também as curvas encontradas através de equações da literatura. Os valores gerados pela equação da literatura 3.4 para aços $\mathrm{C}-\mathrm{Mn}$, ao $\mathrm{Nb}$ e microligados foram colocados na forma das equações 3.6, 3.7 e 3.8, respectivamente. A distribuição em escala logarítmica facilita a visualização.

$$
\begin{aligned}
& \mathrm{Z}=\dot{\varepsilon} \cdot \exp \left(\frac{\mathrm{Q}_{\text {def }}}{\mathrm{RT}}\right) \\
& \sigma_{\mathrm{ss}}=\mathrm{A}_{\mathrm{ss}} \cdot(\mathrm{Z})^{\mathrm{q}}
\end{aligned}
$$

\footnotetext{
* Contribuição técnica ao $69^{\circ}$ Congresso Anual da ABM - Internacional e ao $14^{\circ}$ ENEMET - Encontro Nacional de Estudantes de Engenharia Metalúrgica, de Materiais e de Minas, 21 a 25 de julho de 2014, São Paulo, SP, Brasil.
} 

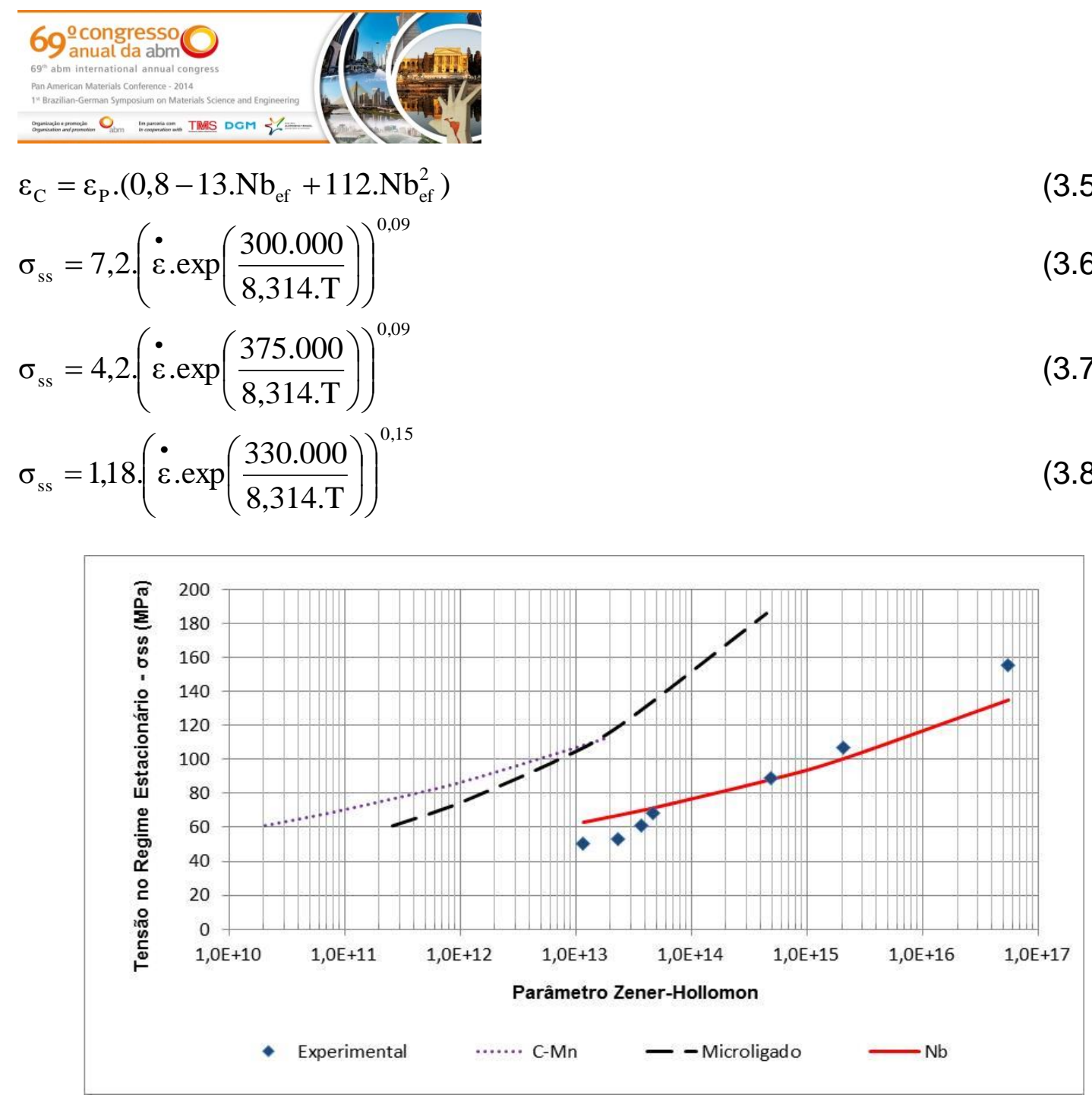

Figura 10 - Tensão no regime estacionário em função do parâmetro Zener-Hollomon. llustrado os pontos extraídos das curvas experimentais dos ensaios isotérmicos e de equações para aços C-Mn (3.6), ao Nb (3.7) e microligados (3.8).

Conforme esperado o modelo para aço ao nióbio foi o que melhor se ajustou ao resultado experimental.

No presente trabalho uma nova equação foi proposta para descrever a tensão no estado estacionário oss para o aço API $5 \mathrm{~L}$ X80. A equação 3.4 foi utilizada como base e as constantes "Ass" e "q" foram obtidas por regressão exponencial em função do parâmetro de Zener-Hollomon (Z) para os dados experimentais, conforme mostrado na Figura 11. O valor das constantes "Ass"e "q" para a nova equação valem 0,94 e 0,13 , respectivamente. Desta forma chegou-se a equação 3.9 que descreve o comportamento do aço em questão.

$\sigma_{\mathrm{ss}}=0,94 \cdot\left(\dot{\varepsilon} \cdot \exp \left(\frac{375 \cdot 000}{8,314 . \mathrm{T}}\right)\right)^{0,13}$

A equação 3.9 foi utilizada para determinar os valores de $\sigma_{s s}$ matematicamente. Estes valores e os resultados experimentais estão dispostos na Figura 11.

\footnotetext{
* Contribuição técnica ao $69^{\circ}$ Congresso Anual da ABM - Internacional e ao $14^{\circ}$ ENEMET - Encontro Nacional de Estudantes de Engenharia Metalúrgica, de Materiais e de Minas, 21 a 25 de julho de 2014, São Paulo, SP, Brasil.
} 

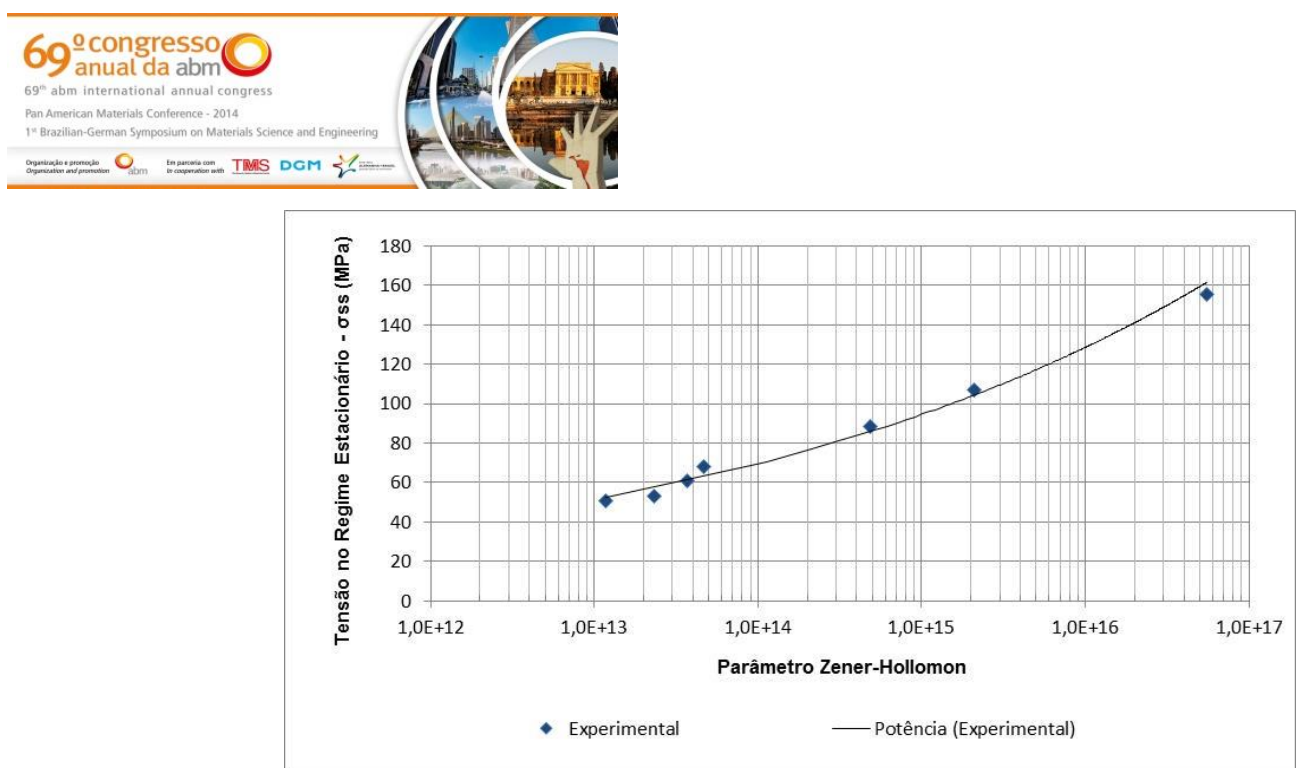

Figura 11 - Tensão no regime estacionário em função do parâmetro Zener-Hollomon. llustrado os pontos extraídos das curvas experimentais dos ensaios isotérmicos e da equação obtida por regressão (3.9).

Comparando as Figuras 10 e 11 observamos que os pontos experimentais se ajustaram melhor no segundo caso.

$\mathrm{Na}$ Tabela 4 podem ser observados os valores da $\varepsilon_{\mathrm{c}}, \varepsilon_{\mathrm{p}}, \varepsilon_{\mathrm{c}} / \varepsilon_{\mathrm{p}}$ e $\sigma_{\mathrm{ss}}$ encontradas experimentalmente e através de equações da literatura $\left(\varepsilon_{c}\right)$ e das equações propostas no presente trabalho ( $\varepsilon_{\mathrm{p}}$ e $\left.\sigma_{\mathrm{ss}}\right)$.

Tabela 4 - Valores de $\varepsilon c$, $\varepsilon p$ e бss obtidos nos ensaios isotérmicos para um aço API $5 \mathrm{~L}$ X80 e através de equações da literatura e de equações propostas no presente trabalho.

\begin{tabular}{|c|c|c|c|c|c|c|c|c|c|}
\hline \multirow[b]{2}{*}{$\mathbf{T}\left({ }^{\circ} \mathbf{C}\right)$} & \multirow[b]{2}{*}{$\dot{\varepsilon}\left(\mathbf{s}^{-1}\right)$} & \multicolumn{4}{|c|}{ Experimentais } & \multicolumn{4}{|c|}{ Modelos Matemáticos para aço ao Nb } \\
\hline & & $\varepsilon_{c}$ & $\varepsilon_{\mathrm{p}}$ & $\varepsilon_{\mathrm{c}} / \varepsilon_{\mathrm{p}}$ & $\begin{array}{c}\sigma_{\mathrm{ss}} \\
(\mathrm{MPa})\end{array}$ & $\begin{array}{c}\varepsilon_{c} \\
(E q .3 .4)\end{array}$ & $\begin{array}{c}\varepsilon_{p} \\
(E q .3 .2)\end{array}$ & $\varepsilon_{\mathrm{c}} / \varepsilon_{\mathrm{p}}$ & $\begin{array}{c}\sigma_{\mathrm{ss}} \\
(\mathrm{MPa}) \\
(\mathrm{Eq} .3 .9)\end{array}$ \\
\hline 1.150 & 0,2 & 0,17 & 0,36 & 0,47 & 50,5 & 0,18 & 0,37 & 0,49 & 52,1 \\
\hline 1.150 & 0,4 & 0,23 & 0,46 & 0,50 & 53,1 & 0,20 & 0,42 & 0,49 & 57,2 \\
\hline 1.150 & 0,8 & 0,35 & 0,62 & 0,57 & 68,3 & 0,23 & 0,47 & 0,49 & 62,7 \\
\hline 1.100 & 0,2 & 0,24 & 0,45 & 0,53 & 61,1 & 0,22 & 0,45 & 0,49 & 60,8 \\
\hline 1.000 & 0,2 & - & 0,70 & - & 88,6 & - & 0,70 & - & 85,8 \\
\hline 950 & 0,2 & - & 0,80 & - & 107,0 & - & 0,89 & - & 104,1 \\
\hline 850 & 0,2 & - & 1,99 & - & 155,6 & - & 1,56 & - & 161,4 \\
\hline
\end{tabular}

Conforme pode ser visto na Tabela 4 os valores da $\varepsilon_{c}$ e $\varepsilon_{p}$ encontrados experimentalmente e através da equação 3.2 estão mais próximos após o ajuste realizado na equação 3.1. Assim como os valores de $\sigma_{\text {ss }}$ calculados através da equação 3.9 ficaram mais próximo do experimental após o ajuste realizado na equação 3.4 .

\section{CONCLUSÃo}

1 - Conforme esperado nos ensaios isotérmicos os valores da tensão de pico aumentam com o decréscimo da temperatura e com o aumento da taxa de deformação, e os valores encontrados para relação $\varepsilon_{c} / \varepsilon_{p}$ foram próximos a 0,5 . As curvas geradas nos ensaios mostram uma similaridade de comportamento com estudos realizados para outros aços.

\footnotetext{
* Contribuição técnica ao $69^{\circ}$ Congresso Anual da ABM - Internacional e ao $14^{\circ}$ ENEMET - Encontro Nacional de Estudantes de Engenharia Metalúrgica, de Materiais e de Minas, 21 a 25 de julho de 2014, São Paulo, SP, Brasil.
} 
2 - Nos ensaios isotérmicos para temperaturas acima da $T_{n r}\left(1.050^{\circ} \mathrm{C}\right)$, o principal mecanismo de restauração é a recristalização dinâmica. Isto pode ser confirmado através da forma da curva de escoamento plástico. Abaixo dessa temperatura as curvas apresentam uma recuperação mais lenta, característica de recuperação dinâmica.

3 - Os valores de $\varepsilon_{c}, \varepsilon_{p}$ e $\sigma_{s s}$ encontrados através de equações da literatura não foram coerentes com os extraídos das curvas dos ensaios isotérmicos. Após ajustes realizado nos modelos para aço com alto $\mathrm{Nb}$ para cálculo da $\varepsilon_{\mathrm{c}}$ e $\sigma_{\mathrm{ss}}$ os valores das equações se aproximaram aos valores obtidos experimentalmente.

\section{REFERENCIAS}

1 Barbosa R. Simulação de processos industriais a partir do ensaio de torção a quente. Belo Horizonte: UFMG; 1989.

2 Regone W. Simulação da laminação a quente de um aço livre de intersticiais (IF) através de ensaio de torção [Tese de Doutorado]. São Paulo: UFSCAR; 2001.

3 Ferraz H. O Aço na Construção Civil. Revista Eletrônica de Ciências. 2005;mar.:15.

4 Barcelos MV. Ensaios de torção a quente em aço estrutural. Vitória: IFES; 2011.

5 Marques A. Avaliação do comportamento mecânico do aço estrutural SAE 4340 através de ensaios de torção a quente. Vitória: IFES - Propemm; 2012.

6 Tasca M. Análise do comportamento mecânico do aço SAE 1518 através de ensaios de torção a quente. Vitória: IFES - Propemm; 2013.

7 Callister Jr. WD. Ciência e engenharia de materiais: Uma introdução. $8^{\circ}$. ed. Rio de Janeiro: LTC; 2012.

8 Padilha AF; Siciliano Jr. F. Encruamento, recristalização, crescimento de grão e textura. 3‥ ed. São Paulo: ABM; 2005.

9 Minami, K. Mathematical modeling of the mean flow stress during the hot strip rolling of Nb steels. ISIJ International. 1996;36(12):1507-1515.

* Contribuição técnica ao $69^{\circ}$ Congresso Anual da ABM - Internacional e ao $14^{\circ}$ ENEMET - Encontro Nacional de Estudantes de Engenharia Metalúrgica, de Materiais e de Minas, 21 a 25 de julho de 2014, São Paulo, SP, Brasil. 